\title{
AVALIAÇÃo dE RISCO À PRODUÇÃO DE FRANGO DE CORTE DO ESTADO DE SÃO PAULO EM FUNÇÃO DA TEMPERATURA AMBIENTE
}

\author{
DOUGLAS D. SALGADO ${ }^{1}$, IRENILZA DE A. NÄÄS ${ }^{2}$
}

\begin{abstract}
RESUMO: As perdas produtivas na avicultura de corte, provenientes de climas com temperaturas diárias elevadas, são potencialmente de grande magnitude, pois abrangem perdas diretas e indiretas. Esta pesquisa teve a finalidade de apontar os municípios localizados onde há mais risco de temperaturas extremas diárias. Os dados históricos meteorológicos de temperaturas diárias, máximas e mínimas, fornecidos pelo CEPAGRI-UNICAMP, foram adaptados para análise estatística, descritiva e exploratória. As temperaturas diárias foram categorizadas em forma binária, estimando-se probabilidades condicionais (riscos) por meio das frequências relativas, obtidas em tabelas de contingência. Utilizando o software Minitab $15^{\circledR}$, foi realizada análise descritiva, análise de risco e, por fim, avaliação de associação. Este trabalho apontou que os municípios da região oeste do Estado de São Paulo foram os mais suscetíveis a apresentarem perda na produção avícola devido às temperaturas ambientais, sendo recomendado maior cuidado com o excesso de calor nos alojamentos das granjas. Também foi constatado que os valores médios e medianos das temperaturas mínimas são bons preditores do risco, devido à alta associação entre o risco e essas variáveis.
\end{abstract}

PALAVRAS-CHAVE: bem-estar animal, temperaturas extremas, risco.

\section{RISK EVALUATION OF BROILER PRODUCTION IN THE STATE OF SÃO PAULO AS FUNCTION OF AMBIENT TEMPERATURE}

\begin{abstract}
Broiler production productive losses due to weather with daily high temperature are potentially of great direct and indirect productive losses. This research aimed to develop a climatologic profile of counties of the State of São Paulo, under the conditions that involved risk of losses in broiler production. A set of historical data given by the CEPAGRI-UNICAMP were adapted to the statistical analysis, and furthermore the descriptive and exploratory analysis were performed with the temperatures. Daily temperature data were categorized in binary form to estimate the conditional probabilities (risk) through the relative frequencies, obtained from tables of contingency. Using the software Minitab $15^{\circledR}$ both descriptive and risk analysis were performed, and at last a connection evaluation. These researches showed that the counties from the West region of the state of São Paulo are the most susceptible to present loss in poultry production due to ambient temperatures. For these counties it is recommended high concern from the producer with the excess of heat inside the houses in the farms. It was also found that mean and median values of minimum temperature are good predictors of risk due to the high association between risk and these variables.
\end{abstract}

KEY WORDS: animal welfare, extreme temperatures, risk.

\section{INTRODUÇÃO}

O desenvolvimento tecnológico brasileiro, voltado à produção zootécnica, vem aperfeiçoando-se ao longo do tempo, equiparando-se aos grandes produtores internacionais, aumentando a produção para exportação, sob os elevados níveis de qualidade exigidos no mercado externo (SILVA, 2000; BRUNOZILLI \& SOUZA, 2004; ROPPA, 2004). Para tanto, justificou-se a otimização do sistema de produção, automação e utilização de recursos de zootecnia de precisão

\footnotetext{
${ }^{1}$ Estatístico, Doutor em Engenharia Agrícola, estatdoug@yahoo.com.br.

${ }^{2}$ Eng $^{\mathrm{a}}$ Civil, Professora Colaboradora, FEAGRI-UNICAMP, irenilza@ feagri.unicamp.br.

Recebido pelo Conselho Editorial em: 20-11-2008

Aprovado pelo Conselho Editorial em: 4-11-2009
} 
(NÄÄS \& CURTO, 2001). Contudo, faz-se também necessária a redução de perdas produtivas, principalmente nas fases finais de criação, sobretudo quando provocada pelo estresse térmico, reduzindo os índices zootécnicos (HAHN, 1982; YAHAV et al., 1995; COOPER \& WASHBURN, 1998; TANKSON et al., 2001). Grande parte do equilíbrio energético na granja de produção de frangos de corte está na manutenção do ambiente interno dentro da faixa de conforto, o que ocorre em função da temperatura ambiente externa ao aviário, além da idade das aves (MACARI \& GONZALES, 1990; DEATON et al., 1997; MOURA, 2001). Sendo a avicultura comercial uma atividade intensiva, as condições adversas de clima podem em parte ser eliminadas ou atenuadas, quando os galpões são localizados em regiões mais favoráveis (ARADAS et al., 2005; MIRAGLIOTTA et al., 2006; VALE et al., 2008).

A temperatura ideal para criação de frangos de corte está próxima aos $20{ }^{\circ} \mathrm{C}$ (MACARI \& GONZALES, 1990; COOPER \& WASHBURN, 1998; TANKSON et al., 2001), a temperatura média da superfície da pele é ao redor de $33{ }^{\circ} \mathrm{C}$, e a temperatura interna, de $41{ }^{\circ} \mathrm{C}$; assim, como existe diferença de temperatura entre a superfície da pele e o ambiente, ocorrerá fluxo de energia (calor) do local mais quente (pele $-33{ }^{\circ} \mathrm{C}$ ) para o mais frio (ambiente - 25 a $28{ }^{\circ} \mathrm{C}$ ), promovendo perda de calor sensível ao ambiente. Estudos dos efeitos de variadas condições térmicas sobre as aves, por meios experimentais controlados, encontraram faixas de alto risco de perda produtiva atingindo até a mortalidade, decorrente, direta e indiretamente, da exposição ao estresse calórico (TANKSON et al., 2001; NIENABER \& HAHN, 2004; PEREIRA, 2005; SALGADO, 2006; VALE et al., 2008).

As análises, descritiva e exploratória, são importantes meios de pré-avaliar os dados de clima, pois fornecem subsídios para o conhecimento mais concreto a respeito das variáveis registradas, o que permite o levantamento de diversas hipóteses importantes para a investigação científica. De maneira geral essas hipóteses podem ser avaliadas por meio de análises confirmatórias, como testes de hipóteses, modelos, etc. (SALGADO, 2006). Estudos de zoneamento de risco têm sido utilizados na agricultura e, na área de climatologia, a aplicação de modelos de predição tem sido dificultada pelas múltiplas interações de fatores ambientais naturais com abruptas variações não lineares, aumentando-se a incerteza sobre a previsibilidade de eventos extremos (JONES, 2003; SCHNEIDER, 2004).

O Brasil possui um rebanho de aproximadamente 822 milhões de aves produtoras de carnes e ovos (IBGE, 2006), cerca de nove milhões de toneladas produzidas por ano, o que faz do País o maior exportador mundial desse alimento. A região Sudeste é a segunda principal região produtora no País, com 28\% de aves alojadas, sobretudo no Estado de São Paulo, com 18\% (UBA, 2008). Nesse cenário, esta pesquisa teve a finalidade de elaborar uma descrição das temperaturas máximas e mínimas em 38 municípios do Estado de São Paulo, avaliar o risco de temperaturas diárias extremas (MACHADO, 1950; SARTORI, 2000 e ROSSATO et al., 2003), apontando-se as localidades que tendem a apresentar condições que são mais propícias às perdas na produção avícola, por excesso de calor, além de associar as medidas estatísticas de descrição ao risco de temperatura extrema.

\section{MATERIAL E MÉTODOS}

Os dados iniciais foram cedidos pelo Centro de Pesquisas Meteorológicas e Climáticas aplicadas à Agricultura da Universidade Estadual de Campinas (CEPAGRI, 2008). Os dados consistiam em temperaturas diárias (temperatura de bulbo seco) máximas e mínimas, registradas por um período de até 17 anos, em 40 estações meteorológicas distribuídas pelo Estado de São Paulo.

\section{Preparação dos dados históricos e classificação das variáveis}

Os dados foram dispostos da seguinte forma na planilha: 1) Nas diferentes colunas, estavam presentes as variáveis de controle - local (V1), dia (V2), mês (V3) e ano (V4), e as variáveis principais - temperatura máxima diária (V5) e temperatura mínima diária (V6), e 2). As linhas 
representam os registros diários consecutivos. Desse modo, a matriz dos dados foi composta por 86 mil registros diários (linhas) e cinco variáveis (colunas). Com base em MACHADO (1950), SARTORI (2000) e ROSSATO et al. (2003), foi elaborada uma variável de classificação binária, denominada "Temperatura diária extrema" (V7). A classificação foi estabelecida a partir das temperaturas máximas e mínimas diárias, simultaneamente, da seguinte maneira, com organização binária: a) Quando a temperatura diária máxima estava acima de $32^{\circ} \mathrm{C}$ e a mínima acima de $22^{\circ} \mathrm{C}$, então a temperatura diária era considerada extrema e recebia o valor "um" (1), e 2) Caso contrário, a temperatura diária era não alarmante, recebendo o valor zero $(0)$.

Foram detectadas as seguintes deficiências nos dados registrados: 1) Falta de sincronismo - os dados não foram registrados para cada cidade a partir do mesmo ano; 2) Desbalanceamento - dados coletados por diferentes períodos, resultando em muitos anos completos de coletas para alguns municípios, enquanto, para outros, não havia mais de um ano completo, e 3) Ausência sistemática de coletas de dados - para alguns anos, não havia registro nos meses finais, ou só existiam nos primeiros meses. Problemas como falta de sincronismo não puderam ser solucionados, pois se fossem aparados o início e o fim do conjunto de dados de períodos diferentes, somente dados de dez municípios seriam aproveitados. Isso corresponderia a uma redução no banco de dados, sobretudo quanto à variabilidade espacial das variáveis no Estado de São Paulo. Logo, sob o ponto de vista dos objetivos deste trabalho, optou-se por manter o maior número de diferentes pontos de registro, mesmo não havendo total sincronismo entre os pontos. Analogamente, pelo mesmo motivo, não se pôde fazer o balanceamento dos registros por município, pois a variação de diferentes períodos de registro era grande. Contudo, foi necessário extrair dados de alguns municípios em que, em determinados anos, havia uma ausência de registros consecutivos. Essa conduta foi necessária para evitar o "vício" nas estimativas. Assim, o banco de dados composto passou a ter dois municípios a menos e, por conseguinte, houve uma ligeira redução de linhas. No total, foram computados 38 municípios com dados diários, contabilizando o total de 77.687 linhas.

\section{Análise descritiva das temperaturas}

As análises inicialmente foram efetuadas com base nas variáveis contínuas, temperaturas mínimas e máximas diárias, para cada município. Assim puderam-se realizar as análises descritivas por meio das medidas de posição: Média, Mediana, Valor máximo, Valor mínimo; e de dispersão: Desvio-Padrão e Erro-Padrão.

\section{Mineração de dados}

Foram realizadas as análises sobre a variável já categorizada, denominada "Temperatura diária extrema", avaliando-se o risco de ocorrência desse fenômeno, para cada município. Por meio da variável V7 (Temperatura Diária Extrema), foi obtida uma tabela de contingência de modo a se mensurarem as probabilidades condicionais, que, por sua vez, foram estimadas pela frequência relativa. As frequências relativas, consequentemente, estimam o risco, pois esse é definido como probabilidade condicional (FLEISS, 2003). Posteriormente, foi avaliada a existência de associação entre as medidas-resumo das temperaturas (mínimas e máximas) e os valores do risco de temperaturas extremas. Para os cálculos de estatísticas descritivas e exploratórias, foi utilizado o software MINITAB $15^{\circledR}$ (MINITAB, 2006).

\section{RESULTADOS E DISCUSSÃO}

\section{Análise das temperaturas mínimas e máximas diárias}

Os resultados das análises descritivas sobre as temperaturas (mínimas, médias e máximas) documentaram resumidamente, as características dos dados, permitindo uma descrição e comparação das temperaturas observadas nos municípios avaliados (Tabelas 1 e 2). A suposição de que as regiões de temperaturas mais elevadas tenderiam a ter maiores índices de risco, tem base em SOARES \& DIAS (1986), que descrevem, em seus resultados, o efeito denominado de persistência térmica temporal, isto é, a probabilidade de ocorrência de calor num determinado dia é maior 
quando houve calor no dia anterior. Assim, a persistência temporal de temperaturas elevadas, em regiões mais quentes, favorece o aumento da frequência de registros de temperaturas extremas diárias, refletindo no aumento do risco.

TABELA 1. Dados descritivos para os municípios, ordenados segundo a média da temperatura mínima diária. Descriptive data for the counties ordered according to the mean daily minimum temperature.

\begin{tabular}{|c|c|c|c|c|c|c|}
\hline $\begin{array}{c}\text { Temperatura Mínima Diária }\left({ }^{\circ} \mathrm{C}\right) \\
\text { Município } \\
\end{array}$ & Média & Erro-Padrão & Desvio-Padrão & Mínimo & Mediana & Máximo \\
\hline Mirante do Paranapanema & 20,23 & 0,15 & 4,17 & 7,00 & 20,00 & 30,00 \\
\hline São José do Rio Preto & 19,47 & 0,10 & 3,72 & 5,00 & 20,00 & 28,00 \\
\hline Araçatuba & 19,17 & 0,06 & 3,57 & 5,00 & 20,00 & 29,00 \\
\hline Osvaldo Cruz & 18,89 & 0,13 & 3,42 & 7,00 & 19,00 & 26,00 \\
\hline Presidente Prudente & 18,73 & 0,05 & 3,74 & 0,00 & 19,40 & 27,70 \\
\hline Tupã & 18,68 & 0,24 & 4,60 & 5,00 & 20,00 & 27,00 \\
\hline Andradina & 18,60 & 0,11 & 3,61 & 0,60 & 19,20 & 26,00 \\
\hline Registro & 18,40 & 0,17 & 3,26 & 10,40 & 18,20 & 25,50 \\
\hline Santos & 18,35 & 0,18 & 3,46 & 9,00 & 18,70 & 26,00 \\
\hline Ubatuba & 18,30 & 0,18 & 3,44 & 7,70 & 19,00 & 25,80 \\
\hline Penápolis & 18,18 & 0,10 & 4,17 & 3,00 & 19,00 & 28,00 \\
\hline Santa Cruz do Rio Pardo & 18,16 & 0,13 & 4,14 & 1,00 & 19,00 & 29,00 \\
\hline Votuporanga & 18,08 & 0,05 & 3,72 & 0,20 & 19,20 & 26,00 \\
\hline Rancharia & 18,05 & 0,13 & 3,49 & 3,00 & 19,00 & 25,00 \\
\hline Tupi Paulista & 17,99 & 0,20 & 3,86 & 2,00 & 19,00 & 24,50 \\
\hline Barretos & 17,55 & 0,06 & 4,19 & $-0,20$ & 19,00 & 27,40 \\
\hline Quatá & 17,14 & 0,20 & 3,83 & 1,00 & 18,00 & 24,00 \\
\hline Sorocaba & 17,06 & 0,13 & 2,39 & 10,00 & 17,00 & 22,80 \\
\hline Matão & 17,04 & 0,14 & 3,79 & $-1,00$ & 18,00 & 26,00 \\
\hline Mococa & 16,93 & 0,05 & 3,48 & 0,00 & 17,80 & 25,00 \\
\hline Paraguaçu Paulista & 16,87 & 0,18 & 4,88 & $-1,00$ & 17,00 & 26,00 \\
\hline Pedrinhas Paulista & 16,83 & 0,18 & 4,80 & $-0,20$ & 17,45 & 27,00 \\
\hline Ribeirão Preto & 16,82 & 0,04 & 3,32 & 0,00 & 17,50 & 24,20 \\
\hline Campinas & 16,64 & 0,05 & 3,36 & 0,30 & 17,20 & 23,40 \\
\hline São Simão & 16,57 & 0,21 & 3,97 & 5,00 & 17,50 & 24,50 \\
\hline Tarumã & 16,52 & 0,23 & 4,37 & 2,00 & 18,00 & 25,00 \\
\hline Tietê & 16,04 & 0,20 & 3,86 & 3,60 & 17,00 & 22,40 \\
\hline Paulínia & 15,98 & 0,23 & 4,29 & 0,00 & 17,00 & 28,00 \\
\hline Taubaté & 15,96 & 0,13 & 4,01 & 4,60 & 16,80 & 24,70 \\
\hline São Carlos & 15,91 & 0,05 & 3,55 & $-2,40$ & 16,50 & 25,40 \\
\hline Taquarituba & 15,90 & 0,06 & 4,18 & $-1,50$ & 16,00 & 26,00 \\
\hline Pindamonhangaba & 15,70 & 0,17 & 4,48 & 0,10 & 16,80 & 28,90 \\
\hline Assis & 15,48 & 0,05 & 4,07 & $-3,20$ & 16,00 & 26,00 \\
\hline Monte Alegre do Sul & 15,35 & 0,14 & 3,75 & $-1,00$ & 16,20 & 21,50 \\
\hline Jundiaí & 15,26 & 0,05 & 3,50 & $-1,00$ & 15,60 & 29,00 \\
\hline Atibaia & 14,96 & 0,07 & 3,62 & 2,10 & 15,60 & 21,60 \\
\hline Piracicaba & 14,91 & 0,15 & 4,17 & 0,00 & 15,50 & 22,80 \\
\hline Piedade & 13,81 & 0,16 & 3,11 & 3,00 & 14,00 & 21,00 \\
\hline
\end{tabular}


TABELA 2. Dados descritivos para os municípios, ordenados segundo a média da temperatura máxima diária. Descriptive data for the counties ordered according to the mean daily maximum temperature.

\begin{tabular}{|c|c|c|c|c|c|c|}
\hline $\begin{array}{l}\text { Temperatura Máxima Diária }\left({ }^{\circ} \mathrm{C}\right) \\
\text { Município }\end{array}$ & Média & Erro-Padrão & Desvio-Padrão & Mínimo & Mediana & Máximo \\
\hline Tupi Paulista & 32,40 & 0,22 & 4,10 & 15,00 & 33,00 & 40,00 \\
\hline Osvaldo Cruz & 31,69 & 0,14 & 3,86 & 11,00 & 32,00 & 40,00 \\
\hline Barretos & 31,53 & 0,05 & 3,68 & 14,80 & 31,60 & 42,00 \\
\hline Rancharia & 30,91 & 0,13 & 3,62 & 12,00 & 32,00 & 40,00 \\
\hline Pedrinhas Paulista & 30,89 & 0,16 & 4,43 & 13,00 & 31,40 & 40,00 \\
\hline São José do Rio Preto & 30,75 & 0,11 & 4,08 & 12,00 & 31,00 & 41,00 \\
\hline Quatá & 30,66 & 0,26 & 5,01 & 11,00 & 31,00 & 39,00 \\
\hline Penápolis & 30,62 & 0,10 & 4,18 & 11,00 & 32,00 & 41,00 \\
\hline Votuporanga & 30,54 & 0,05 & 3,65 & 5,80 & 31,00 & 40,40 \\
\hline Andradina & 30,36 & 0,11 & 3,74 & 11,90 & 31,00 & 40,00 \\
\hline Araçatuba & 30,19 & 0,07 & 4,04 & 15,00 & 31,00 & 42,00 \\
\hline Paraguaçu Paulista & 29,98 & 0,16 & 4,20 & 7,00 & 31,00 & 40,00 \\
\hline Paulínia & 29,87 & 0,19 & 3,59 & 15,00 & 30,00 & 37,00 \\
\hline Pindamonhangaba & 29,82 & 0,16 & 4,36 & 17,80 & 30,20 & 38,40 \\
\hline Tupã & 29,64 & 0,24 & 4,53 & 15,00 & 30,00 & 38,00 \\
\hline São Simão & 29,43 & 0,19 & 3,64 & 17,00 & 29,80 & 39,00 \\
\hline Santa Cruz do Rio Pardo & 29,28 & 0,14 & 4,61 & 10,00 & 29,60 & 38,80 \\
\hline Matão & 29,25 & 0,14 & 3,75 & 13,00 & 29,50 & 37,50 \\
\hline Presidente Prudente & 29,25 & 0,05 & 3,81 & 0,00 & 29,70 & 38,80 \\
\hline Tarumã & 29,24 & 0,23 & 4,45 & 13,00 & 30,00 & 37,60 \\
\hline Ribeirão Preto & 29,08 & 0,04 & 3,28 & 12,20 & 29,40 & 38,80 \\
\hline Mococa & 28,78 & 0,05 & 3,30 & 13,20 & 29,00 & 37,80 \\
\hline Assis & 28,48 & 0,05 & 4,01 & 0,00 & 29,00 & 38,70 \\
\hline Mirante do Paranapanema & 28,25 & 0,12 & 3,25 & 15,00 & 29,00 & 38,00 \\
\hline Piracicaba & 28,21 & 0,13 & 3,52 & 15,20 & 28,80 & 35,40 \\
\hline Tietê & 27,93 & 0,21 & 3,97 & 14,40 & 28,30 & 36,30 \\
\hline Taubaté & 27,88 & 0,14 & 4,17 & 17,00 & 28,00 & 37,80 \\
\hline Campinas & 27,85 & 0,05 & 3,64 & 12,40 & 28,20 & 36,80 \\
\hline Ubatuba & 27,66 & 0,22 & 4,17 & 15,80 & 28,00 & 38,40 \\
\hline Sorocaba & 27,64 & 0,17 & 3,27 & 18,00 & 28,00 & 35,00 \\
\hline Santos & 27,62 & 0,19 & 3,67 & 17,80 & 27,50 & 40,80 \\
\hline Monte Alegre do Sul & 27,52 & 0,14 & 3,77 & 12,20 & 27,80 & 36,20 \\
\hline Jundiaí & 27,34 & 0,06 & 3,96 & 12,40 & 27,80 & 36,60 \\
\hline São Carlos & 27,31 & 0,05 & 3,52 & 10,60 & 27,70 & 38,80 \\
\hline Registro & 27,06 & 0,27 & 5,11 & 13,80 & 27,00 & 38,40 \\
\hline Taquarituba & 26,55 & 0,07 & 4,56 & 10,60 & 27,00 & 37,00 \\
\hline Atibaia & 26,20 & 0,08 & 4,11 & 0,00 & 26,60 & 35,60 \\
\hline Piedade & 22,09 & 0,23 & 4,46 & 10,00 & 22,00 & 34,00 \\
\hline
\end{tabular}


Os municípios de Mirante do Paranapanema e São José do Rio Preto foram aqueles com temperaturas mínimas em patamares mais elevados, visto que as médias das temperaturas mínimas já alcançaram valores próximos de uma temperatura mínima de alerta (MACHADO, 1950). Por outro lado, o município de Piedade foi aquele que apresentou a menor média das temperaturas mínimas entre todas as cidades estudadas, sendo menor a possibilidade de ocorrência de um dia de temperatura extrema.

Considerada a referência de temperatura máxima de $32{ }^{\circ} \mathrm{C}$ (MACHADO, 1950; SARTORI, 2000 e ROSSATO et al., 2003), foi possível verificar que os municípios de Barretos e Tupi Paulista são passíveis de alcançar temperaturas máximas diárias críticas, que afetam negativamente o desempenho produtivo das aves (DEATON et al., 1997; ARADAS et al., 2005; MIRAGLIOTTA et al., 2006 e VALE et al., 2008). Contudo, na região onde se localiza o município de Piedade, houve menor valor médio de temperatura máxima, sugerindo que, nessa região, não haverá risco de ocorrência de temperatura extrema.

\section{Análise do risco de temperatura extrema diária}

Os riscos foram obtidos pela estimativa das probabilidades condicionais e foram calculados a partir de uma tabela de contingência, que foi configurada especialmente para a avaliação e comparação desses valores. A obtenção da tabela de contingência foi proveniente de um cruzamento entre duas variáveis categóricas que possuíam diferentes classes (FLEISS, 2003). A Tabela 3 apresenta os riscos de ocorrência de temperaturas extremas, dado um determinado município. Verifica-se que Andradina possui um risco de 11,22\%, ou seja, valor que corresponde à probabilidade de haver, em qualquer dia, uma temperatura extrema nesse município. Foram encontrados os seguintes municípios com maior risco de incidência de temperatura extrema diária, que provocaria problemas de estresse térmico na produção de frangos de corte, conforme indicam YAHAV et al. (1995) e COOPER \& WASHBURN (1998): Tupã (29,04\%), Araçatuba (23,06\%), São José do Rio Preto (21,90\%), Osvaldo Cruz (19,84\%) e Penápolis (19,28\%). Também foram apontados os municípios de risco zero (0,00\%): Atibaia, Monte Alegre do Sul e Piedade.

Comparando-se as Tabelas 1; 2 e 3, constatou-se que a maioria dos municípios que possuem temperaturas mais elevadas, pertence à região oeste do Estado de São Paulo. Verificou-se também que a classificação obtida pelos riscos de temperaturas extremas está mais vinculada com o perfil da temperatura mínima, do que o da temperatura máxima.

Os municípios de Araçatuba e de São José do Rio Preto foram os que apresentaram temperaturas ambientais extremas mais altas e com elevados riscos de temperaturas extremas diárias, o que os torna vulneráveis ao impacto negativo do efeito térmico sobre a criação de aves, de acordo com o preconizado por DEATON et al.(1997), MIRAGLIOTTA et al. (2006) e VALE et al. (2008). Nesse contexto, é dada maior atenção à região de São José do Rio Preto, pois é um dos municípios de intensa produção avícola de corte (APA, 2008). Assim, os produtores devem ter mais investimentos e alternativas para mitigar as elevadas temperaturas ambientais, às quais seus alojamentos estão submetidos.

As evidências da relação entre determinadas características das temperaturas e os riscos de temperaturas extremas foram encontradas por meio da análise de Componentes Principais (Figura 1). Essa análise permitiu a visualização conjunta da relação entre as medidas estatísticas das temperaturas e o risco de temperaturas extremas. As medidas referentes às temperaturas mínimas estão acompanhadas de "(T-mín)", enquanto aquelas referentes às temperaturas máximas estão acompanhadas de "(T-máx)”. Associações dessa natureza foram encontradas por PEREIRA (2005) e SALGADO (2006), quando utilizaram metodologia semelhante. 
TABELA 3. Distribuição percentual de ocorrência de temperatura extrema por município, ordenada pelo risco. Percentage distribution of extreme temperature occurrence by county ordered according to the risk.

\begin{tabular}{|c|c|c|c|}
\hline \multirow{2}{*}{ Município } & \multicolumn{3}{|c|}{ Temperatura Extrema (\%) } \\
\hline & Ausente & Presente & Total \\
\hline Tupã & 70,96 & 29,04 & 100 \\
\hline Araçatuba & 76,94 & 23,06 & 100 \\
\hline São José do Rio Preto & 78,10 & 21,90 & 100 \\
\hline Osvaldo Cruz & 80,16 & 19,84 & 100 \\
\hline Penápolis & 80,72 & 19,28 & 100 \\
\hline Santa Cruz do Rio Pardo & 84,57 & 15,43 & 100 \\
\hline Rancharia & 87,00 & 13,00 & 100 \\
\hline Presidente Prudente & 87,22 & 12,78 & 100 \\
\hline Paraguaçu Paulista & 88,10 & 11,90 & 100 \\
\hline Tupi Paulista & 88,22 & 11,78 & 100 \\
\hline Andradina & 88,78 & 11,22 & 100 \\
\hline Mirante do Paranapanema & 89,60 & 10,40 & 100 \\
\hline Barretos & 91,21 & 8,79 & 100 \\
\hline Pedrinhas Paulista & 92,05 & 7,95 & 100 \\
\hline Registro & 92,33 & 7,67 & 100 \\
\hline Votuporanga & 94,39 & 5,61 & 100 \\
\hline Santos & 94,52 & 5,48 & 100 \\
\hline Ubatuba & 94,52 & 5,48 & 100 \\
\hline Quatá & 96,16 & 3,84 & 100 \\
\hline Taquarituba & 96,46 & 3,54 & 100 \\
\hline Tarumã & 96,71 & 3,29 & 100 \\
\hline Matão & 97,31 & 2,69 & 100 \\
\hline Sorocaba & 97,81 & 2,19 & 100 \\
\hline Taubaté & 99,03 & 0,97 & 100 \\
\hline Pindamonhangaba & 99,04 & 0,96 & 100 \\
\hline Mococa & 99,08 & 0,92 & 100 \\
\hline Paulínia & 99,18 & 0,82 & 100 \\
\hline São Simão & 99,18 & 0,82 & 100 \\
\hline Campinas & 99,25 & 0,75 & 100 \\
\hline Tietê & 99,44 & 0,56 & 100 \\
\hline São Carlos & 99,52 & 0,48 & 100 \\
\hline Assis & 99,54 & 0,46 & 100 \\
\hline Ribeirão Preto & 99,59 & 0,41 & 100 \\
\hline Piracicaba & 99,86 & 0,14 & 100 \\
\hline Jundiaí & 99,92 & 0,08 & 100 \\
\hline Atibaia & 100,00 & 0,00 & 100 \\
\hline Monte Alegre do Sul & 100,00 & 0,00 & 100 \\
\hline Piedade & 100,00 & 0,00 & 100 \\
\hline Total & 94,30 & 5,70 & 100 \\
\hline
\end{tabular}




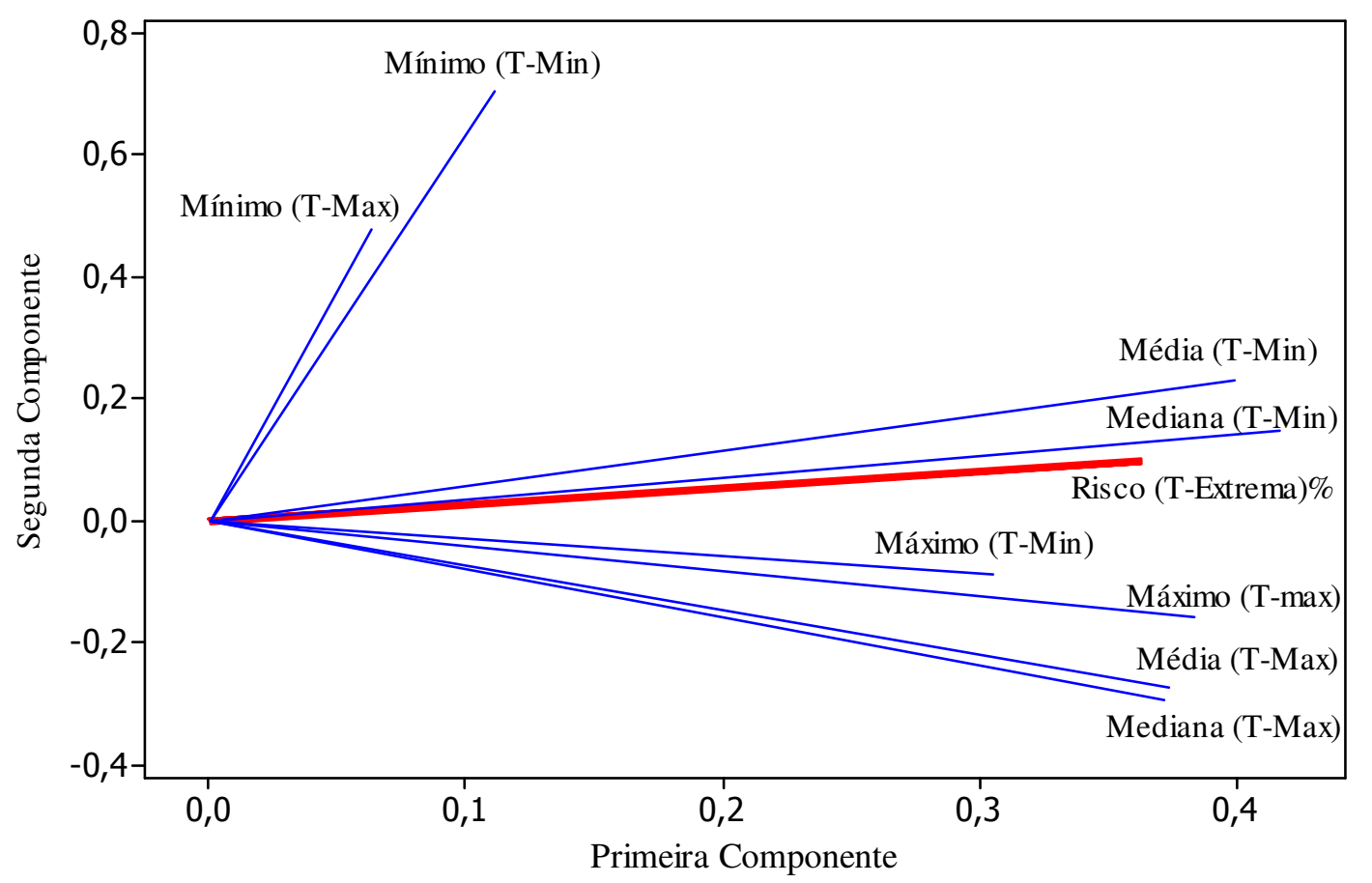

FIGURA 1. Gráfico de Componentes Principais dos riscos de incidência de temperaturas extremas para avicultura. Graph of Principal Components of the extreme temperatures incidence risk for poultry production.

\section{CONCLUSÕES}

A partir de dados meteorológicos históricos, foi possível apontar as regiões onde se localizam os municípios do Estado de São Paulo com maiores temperaturas (mínimas e máximas), bem como calcular o risco de ocorrência de temperaturas extremas. Fazendo-se um comparativo dos resultados, foram destacados os municípios de Araçatuba, Osvaldo Cruz, Mirante do Paranapanema, São José do Rio Preto e Tupã. Dentre as regiões de maior risco à ocorrência de temperaturas diárias extremas, destaca-se a de São José do Rio Preto (aproximadamente 22\% de risco), que é de intensa produção avícola. Verificou-se que a média e, principalmente, as medianas das temperaturas mínimas têm forte relação com os valores encontrados de risco de temperaturas extremas, isso pode resultar em diminuição em custos operacionais e computacionais quando se deseja estimar esse risco.

\section{REFERÊNCIAS}

APA. ASSOCIAÇÃO PAULISTA DOS AVICULTORES. Municípios de intensa produção avícola no Estado de São Paulo. Disponível em: <http://www.apa.com.br>. Acesso em: 15 fev. 2008.

ARADAS, M.E.C.; NÄÄS, I.A.; SALGADO, D.D. Comparing the thermal environment in broiler houses using two bird densities under tropical conditions. CIGR E-Journal, College Station, v.7, p.1-12, 2005.

BRUNOZILLI, J.; SOUZA, D.P. Produtividade regional. Avicultura Industrial, Itu, v.1119, n.2, p.22-24, 2004.

CEPAGRI. CENTRO DE PESQUISAS METEOROLÓGICAS E CLIMÁTICAS APLICADAS À AGRICULTURA. Clima das cidades do Estado de São Paulo. Disponível em: <http://www.cpa. unicamp.br/produção/trabalhos-em-anais-de-eventos/2003/tema1_2.pdf/download.pdf>. Acesso em: 24 set. 2008. 
COOPER, M.A.; WASHBURN, K.W. The relationships of body temperature to weight gain, feed consumption, and feed utilization in broilers under heat stress. Poultry Science, Stanford, v.77, n.2, p.237-242, 1998.

DEATON, J.W.; REECE, F.N.; MCNAUGHTON, N. The effect of temperature during the growing period on broiler performance. Poultry Science, Ithaca, v.57, n.1, p.1.070-1.074, 1997.

FLEISS, J.L.; LEVIN, B.; PAIK, M.C. Statistical methods for rates and proportions. New York: John Wiley \& Sons, 2003. 328 p. (Series in Probability and Statistics)

HAHN, G.L. Compensatory performance in livestock: influences on environmental criteria. In: INTERNATIONAL LIVESTOCK ENVIRONMENT SYMPOSIUM, 2., 1982. Proceedings... St. Joseph: ASABE, 1982. p.285-294.

IBGE. INSTITUTO BRASILEIRO DE GEOGRAFIA E ESTATÍSTICA. Produção agropecuária 2006. Disponível em: <http://www.ibge.gov.br/home/presidencia/noticias/21052004 biomashtml. shtm>. Acesso em: 10 jan. 2008.

JONES, R. Managing climate risks. In: OECD WORKSHOP ON THE BENEFITS OF CLIMATE CHANGE: IMPROVING INFORMATION FOR POLICY MAKERS, 1., 2003, Paris.

Proceedings... Paris: OECD, 2003. p.37.

MACARI, M.; GONZALES, E. Fisiopatogenia da síndrome da morte súbita em frangos de corte. In: CONFERÊNCIA APINCO DE CIÊNCIA E TECNOLOGIA AVÍCOLAS, 1990, Campinas. Anais... Campinas: APINCO, 1990. p.65-73.

MACHADO, F.P. Contribuição ao estudo do clima do Rio Grande do Sul. Rio de Janeiro: IBGE, 1950. p.91.

MINITAB INC. Minitab ${ }^{\circledR}$ 15.1.0.0. State College, 2006.

MIRAGLIOTTA, M.Y.; NÄÄS, I. de A.; MANZIONE, R.L.; NASCIMENTO, F.F. Spatial analysis of stress conditions inside broiler house under tunnel ventilation. Scientia Agricola, Piracicaba, v.63, n.5, p.426-432, 2006.

MOURA, D.J. Ambiência na produção de aves de corte In: SILVA, I.J.O. Ambiência na produção de aves em clima tropical. Jaboticabal: Associação Brasileira de Engenharia Agrícola, 2001. v.2, p.185-198. (Série Engenharia Agrícola)

NÄÄS, I.A.; CURTO, F.P. Avicultura de precisão. In: DA SILVA, I. J. O. Ambiência na produção de aves em clima tropical. Jaboticabal: Associação Brasileira de Engenharia Agrícola, 2001. v.2, p.1-30. (Série Engenharia Agrícola)

NIENABER, J.A.; HAHN, G.L. Engineering and management practices to ameliorate livestock heat stress. In: INTERNATIONAL SYMPOSIUM OF THE CIGR. NEW TRENDS IN FARM BUILDINGS, 1., 2004, Évora. Proceedings... Évora: CIGR, 2004. 1 CD-ROM.

PEREIRA, D.F. Metodologia para estimativa de bem-estar de matrizes de frango de corte, utilizando monitoramento digital e construção de modelos de simulação. $2005.155 \mathrm{f}$. Tese (Doutorado em Construções Rurais e Ambiência) - Faculdade de Engenharia Agrícola, Universidade Estadual de Campinas, Campinas, 2005.

ROPPA, L. Evolução do plantel total de suínos. Disponível em: <http://www. porkworld.com.br.> Acesso em: 30 mar. 2004.

ROSSATO, P.S.; SARTORI, M.G.B.; MISSIO, L.R. As ondas de calor na região central do RS entre os meses de maio a outubro. In: SIMPÓSIO BRASILEIRO DE GEOGRAFIA FÍSICA APLICADA, 10., 2003, Rio de Janeiro. Anais... Rio de Janeiro: DGFA, 2003. 1 CD-ROM.

SALGADO, D.D. Modelo estatístico para predição de bem-estar de reprodutoras de frango de corte baseado em dados de ambiente e análise do comportamento. 2006.126 f. Dissertação 
(Mestrado em Construções Rurais e Ambiência) - Faculdade de Engenharia Agrícola, Universidade Estadual de Campinas, Campinas, 2006.

SARTORI, M.G.B. Clima e percepção. 2000. 138 f. Tese (Doutorado) - Faculdade de Filosofia, Letras e Ciências Humanas, Universidade de São Paulo, São Paulo, 2000.

SCHNEIDER, S.H. Abrupt non-linear climate change, irreversibility and surprise. Global Environmental Change, East Anglia, v.14, n.1, p.245-258, 2004.

SILVA, R.G. Introdução à bioclimatologia animal. São Paulo: Nobel, 2000. 285 p.

SOARES, J.R.; DIAS, M.A.F.S. Probabilidade de ocorrência de alguns eventos meteorológicos extremos na cidade de São Paulo. Revista Brasileira de Meteorologia, Rio de Janeiro, v.1, n.2, p.67-75, 1986.

TANKSON, J.D.; VIZZIER-THAXTON, Y.; THAXTON, J.P.; MAY, J.D.; CAMERON, J.A. Stress and nutritional quality of broilers. Poultry Science, Stanford, v.80, n.9, p.1.384-1.389, 2001. UBA. UNIÃO BRASILEIRA DE AVICULTURA. Relatório 2007-2008. Disponível em: $<$ http://www.uba.org.br/index.htm>. Acesso em: 20 set. 2008.

VALE, M.M.; MOURA, D.J.; NÄÄS, I.A.; OLIVEIRA, S.R.M.; RODRIGUES, L.H.A. Data mining to estimate broiler mortality when exposed to heat wave. Scientia Agricola, Piracicaba, v.65, n.3, p.223-229, 2008.

YAHAV, S.; GOLDFELD, S.; PLAVNIK, I.; HURWITZ, S. Physiological response of chickens and turkeys to relative humidity during exposure to high ambient temperature. Journal of Thermal Biology, Durham, v.20, n.3, p.245-253, 1995. 